\title{
Wind Speed Forecasting Using LSSVM Model Based On a Novel Optimization Algorithm
}

\author{
Yang Bai ${ }^{1}$, Jianyan Tian ${ }^{1,}$, Fang Wang ${ }^{1}$, Wei Gao ${ }^{2}$, Shengqiang Yang ${ }^{2}$ and Xiaoyang Liu ${ }^{1}$ \\ ${ }^{1}$ College of Information Engineering, Taiyuan University of Technology, Taiyuan 030024, China \\ ${ }^{2}$ College of Mechanical Engineering, Taiyuan University of Technology, Taiyuan 030024, China \\ ${ }^{*}$ Corresponding author
}

\begin{abstract}
Wind speed forecasting has significant influence on wind energy development. In the paper, a least square support vector machine with a novel optimization algorithm was used to improve the performances of wind speed forecasting model. Coupled simulated annealing (CSA) and simplex algorithm were combined in the novel optimization algorithm to optimize parameters of LSSVM forecasting model. Firstly, parameters were optimized by CSA in global scope. Then, parameters which got from CSA were optimized by simplex algorithm to get the best parameters. Finally, the LSSVM model with best parameters was applied to wind speed forecasting. Based on the data obtained from a wind farm in Shanxi province, the simulation results show that comparing with the support vector machine (SVM) model with grid-search and the LSSVM model with particle swarm optimization, the proposed model has better performances on accuracy and training time, thereby it helps make reasonable decisions for power scheduling and dispatch.
\end{abstract}

Keywords-wind speed forecasting; least squares support vector machine (LSSVM); coupled simulated annealing algorithm; simplex algorithm

\section{INTRODUCTION}

Wind energy, as a kind of renewable energy sources, plays an important role in power system. It is also an important component of the clean energy. Wind power has become the third largest energy in China, following behind thermal power and hydroelectric power. As known as, wind power relies heavily on wind energy. However, the characteristics of wind energy such as intermittence, fluctuation and unsteadiness, imply that the wind power has similar features with wind energy [1]. Therefore, the grid integration problems of wind farm, such as system reliability and power quality will come into questions. Wind speed forecasting is one of the methods to solve these issues.

In the last decade, people focused more on how to improve the forecasting accuracy of wind speed and wind power. And lots of forecasting models are proposed such as time series model, artificial neural network and support vector machine (SVM). Salcedo-Sanz et al [2] proposed an evolutionary support vector regression algorithms for wind speed forecasting. Moreover, lots of improvements around these algorithms are studied to improve the forecasting accuracy. Among these individual models, SVM appears to be more effective for its characteristics, such as fixed structure and strong generalization ability [3]. It has been proved that the support vector machine has a good performance in forecasting field [4]. As a further study, Suykens et al [5] proposed the least square support vector machine (LSSVM) based on SVM, which decreased computation complexity. In addition, LSSVM is widely used to solve classification and regression problems [6]. Therefore, LSSVM can be used to forecast wind speed based on its significant advantages.

The parametric problem is a main issue for the application of LSSVM. At the same time, people are greatly interested in the methods about how to get proper parameters. Many techniques such as grid-search, genetic algorithm and particle swarm optimization algorithm are proposed. However, when large-scale data are applied to train the model, the training process of these algorithms will be time-consuming. According to this situation, LSSVM based on coupled simulated annealing and simplex algorithm is proposed to forecast wind speed. It combines the advantages of global search and local search to improve the forecasting accuracy and the training speed.

\section{LSSVM MODEL BASED ON A NOVEL OPTIMIZATION ALGORITHM}

\section{A. The Principle of Least Square Support Vector Machine}

The structure of least square support vector machine is based on VC dimension theory and structural risk minimization principle. Classical squared loss function and equality constraints are two big features for the structure of LSSVM algorithm. Since forecasting problems belong to the regression field, the algorithm of LSSVM in regression filed can be introduced as follows [7].

Assume the training set is $\mathrm{T}=\left\{\left(\boldsymbol{X}_{1}, y_{1}\right), \ldots, \quad\left(\boldsymbol{X}_{\mathrm{i}}, y_{\mathrm{i}}\right), \ldots\right.$, $\left.\left(\boldsymbol{X}_{\mathrm{n}}, y_{\mathrm{n}}\right)\right\}$, where $\boldsymbol{X}_{\mathrm{i}} \in \mathrm{R}^{p}$ and $p$ represents the dimension of input variables; $y_{i}$ represents the output variable, $i=1, \ldots, n$.

Then, the regression function can be defined as follows:

$$
y=\boldsymbol{w}^{\mathrm{T}} \varphi(\boldsymbol{X})+b
$$

where $\boldsymbol{w}$ represents weight vector; $\boldsymbol{b}$ represents the bias value; $\varphi(\boldsymbol{X})$ represents the mapping function. The function should be solved through the training data. The solution of the problem can be transformed into the following equations:

$$
\min _{w, b, \varepsilon} J(\boldsymbol{w}, \varepsilon)=\frac{1}{2}\|\boldsymbol{w}\|^{2}+\frac{C}{2} \sum_{i=1}^{n} \varepsilon_{i}^{2}
$$




$$
\text { s.t. } \quad y_{i}=\left(\boldsymbol{w}^{\mathrm{T}} \varphi\left(\boldsymbol{X}_{i}\right)+b\right)+\varepsilon_{i}
$$

where $C$ represents regularization parameter; $\varepsilon_{i}$ represents error band.

Then a function can be derived by introducing Lagrange multiplier, as follows:

$$
L(\boldsymbol{w}, b, e ; \alpha)=J(\boldsymbol{w}, e)-\sum_{i=1}^{n} \alpha_{i}\left\{\boldsymbol{w}^{\mathrm{T}} \cdot \varphi\left(\boldsymbol{X}_{i}\right)+b+\varepsilon_{i}-y_{i}\right\}
$$

where $\alpha_{i}$ represents the Lagrange multiplier.

Through a series of solving process, equation (5) can be derived as follows:

$$
f(\boldsymbol{X})=\sum_{i=1}^{n} \alpha_{i} k\left(\boldsymbol{X}_{i}, \boldsymbol{X}\right)+b
$$

Radial basis kernel is applied in the algorithm of LSSVM because of its superior learning ability [8]. The concrete form of radial basis kernel is as follows:

$$
\exp \left(-\left\|\boldsymbol{x}-\boldsymbol{x}_{\boldsymbol{k}}\right\|^{2} / 2 \sigma^{2}\right)
$$

In the derivation process, regularization parameter $C$ and kernel parameter $\sigma$ are unknown variables, which are important factors to a better forecasting. Therefore, a proper algorithm is required to get the best parameters for the regression function, that is, the LSSVM algorithm.

\section{B. The novel Optimization Algorithm for Parameter Optimization}

Xavier-de-souza et al [9] proposed the coupled simulated annealing (CSA) algorithm. The principle is that the multiple simulated annealing algorithms are computed in the parallel to get the best parameters. Moreover, the acceptance probability function consists of all energy information of each simulated annealing algorithm. Global search, fast searching speed and less sensitive to initial parameters [9] are the characteristics of the algorithm. The object of simplex algorithm is to find the optimal point through reflection, contraction, extension et al operations to replace the best point in simplex [10]. The simplex algorithm has a superior performance on local search, but it is sensitive for initial parameters [11].

Hence, the CSA algorithm can be used to search parameters in global scope. The simplex algorithm is used to optimize the parameters from the CSA algorithm and form the complementary advantages at the same time. Therefore, the novel optimization algorithm combining with CSA and simplex can have a good performance on parameter optimization for LSSVM. The detailed process of parameter optimizing can be shown in Figure 1.

The initial solution of set $X$ consists of multiple sets of parameters and each set of parameters includes regularization parameter $C$ and kernel parameter $\sigma$. If the number of simulated annealing is $m$, then $m$ sets of parameters are included in the set $X$. And several acceptance probability functions can be selected in the CSA algorithm. Coupling term $(\gamma)$ including all information of energy function's $(E(x))$, acceptance probability function $\left(P_{i}\right)$, acceptance $\left(T_{k}^{a c}\right)$ schedule and generation $\left(T_{k}\right)$ schedule are selected according to the reference [9].

\section{CASE STUDY}

\section{A. Wind Speed Forecasting Model}

The data are gathered from a wind tower of a wind farm in Shanxi province. The data consist of the historic values of wind speed, wind direction, temperature and humidity. The time interval is 30 minutes. In some degrees, wind speed alterations can be affected by meteorological factors such as temperature, humidity and wind direction. Therefore, these meteorological factors are taken into account when building wind speed forecasting model. Data preprocessing is necessary to avoid the disadvantages caused by their units. Herein, Zscore standard is used to process the data. Through our study based on the practical data gathered from a wind farm in Shanxi province, it is found that the better forecasting result will be got when the one prior moment wind speed point, wind direction point, temperature point and humidity point are taken as input variables. Moreover, the root of mean square error (RMSE), mean absolute error (MSE), and mean absolute percentage error (MAPE) are used to evaluate the forecasting results.

\section{B. Simulation Results and Analysis}

Training samples are needed to build the LSSVM forecasting model in the forecasting process. Different sizes of training samples may result in different parameters for LSSVM forecasting model, and can causes different forecasting results. Therefore, ensuring a proper number of training samples is a significant step of the final forecasting result. Different sizes of samples are used to train LSSVM model such as $120,240,360,480,600,720,840,960,1080$ and 1200. 200 fixed data points are used to validate the performances of LSSVM model. The error criterion, $M A E$, is selected to evaluate performances with different training samples. And the result is shown in Table 1. It shows that the forecasting accuracy has a rising trend along with the increasing training samples although some fluctuations are occurred. Moreover, the best performance can be obtained when using 960 training samples in the simulation. LSSVMlab toolbox is used in the simulation process.

In order to compare the performances of the adopted algorithm, the values of forecasting error from M1 and M2 models is shown in Table 2, where M1 represents the LSSVM forecasting model using CSA and simplex algorithm; M2 represents the LSSVM forecasting model only using CSA. It is shown that the LSSVM forecasting model has a better performance when using CSA and simplex algorithm.

The SVM forecasting model with grid-search (M3) and LSSVM forecasting model with particle swarm algorithm (M4) are used to compare with M1, to further verify the performance of M1. In M3, the search range of regularization parameter $C$ and kernel parameter $\sigma$ is $\left[2^{-5}, 2^{5}\right]$ and $\left[2^{-5}, 2^{5}\right]$, and the step is $2^{1}$. In M4, the size of initial particles is 10 , the maximum iterative number is 100 , the cognitive learning 
factor is 1.5 , and social learning factor is 1.7. In the data set, the size of training sample is 960 and the size of testing samples is 50. Every training time of models were obtained from a PC with Intel Core I-7 3770 3GB of RAM. The values of forecasting error indexes are shown in Table 3. Forecasting values and real values are shown in Figure 2. It is shown in Table 3 that M1 has the highest forecasting accuracy. The accuracy of M1 in $M A E$ improves by $10.7 \%$ when compare with M4, and it improves by $27.7 \%$ when compared with M3. In $M A P E$, it improves by $7.5 \%$ when compare with M4, and it improves by $22.8 \%$ when compared with M3. And in RMSE it improves by $10.3 \%$ when compare with M4, and it improves by $24.6 \%$ when compared with M3. It is easy to find that the training time of $\mathrm{M} 1$ is significantly shorter than other two models. Moreover, the Figure 2 shows that M1 has the best



performance of tracking wind speed, and M3 and M4 have poor performances in tracking wind speed among some testing data points.

TABLE I. FORECASTING ERRORS FOR DIFFERENT TRAINING SAMPLES.

\begin{tabular}{lccccc}
\hline Error criterion & 120 & 240 & 360 & 480 & 600 \\
\hline$M A E(\mathrm{~m} / \mathrm{s})$ & 2.69 & 3.19 & 1.15 & 1.48 & 1.77 \\
& & & & & \\
\hline Error criterion & 720 & 840 & 960 & 1080 & 1200 \\
\hline$M A E(\mathrm{~m} / \mathrm{s})$ & 1.44 & 1.11 & 0.85 & 0.98 & 0.89 \\
\hline
\end{tabular}

FIGURE I. THE FLOW CHART OF PARAMETERS OPTIMIZATION.

TABLE II. FOECASTING ERROR INDEXES FOR M1 AND M2.

\begin{tabular}{cllc}
\hline Model & MAE $(\mathrm{m} / \mathrm{s})$ & MAPE $(\%)$ & $R M S E(\mathrm{~m} / \mathrm{s})$ \\
\hline M1 & 0.7149 & $7.67 \%$ & 0.912 \\
M2 & 0.7601 & $7.94 \%$ & 0.982 \\
\hline
\end{tabular}

TANLE III. FORECASTING ERROR INDEXS FOR THREE FORECASTING MODELS.

\begin{tabular}{ccccc}
\hline Model & MAE $(\mathrm{m} / \mathrm{s})$ & MAPE $(\%)$ & $R M S E(\mathrm{~m} / \mathrm{s})$ & $\operatorname{TIME}(\mathrm{s})$ \\
\hline M1 & 0.7149 & $7.67 \%$ & 0.9120 & 13.87 \\
M3 & 0.9893 & $9.94 \%$ & 1.2101 & 67.36 \\
M4 & 0.8011 & $8.29 \%$ & 1.0171 & 215.75 \\
\hline
\end{tabular}




\section{CONCLUSION}

In order to further improve the performances of LSSVM wind speed forecasting model, a LSSVM model with coupled simulated annealing and simplex algorithm is applied in wind speed forecasting model in this paper. The data obtained from a wind farm in Shanxi province have been used to test the accuracy and efficiency of this model.

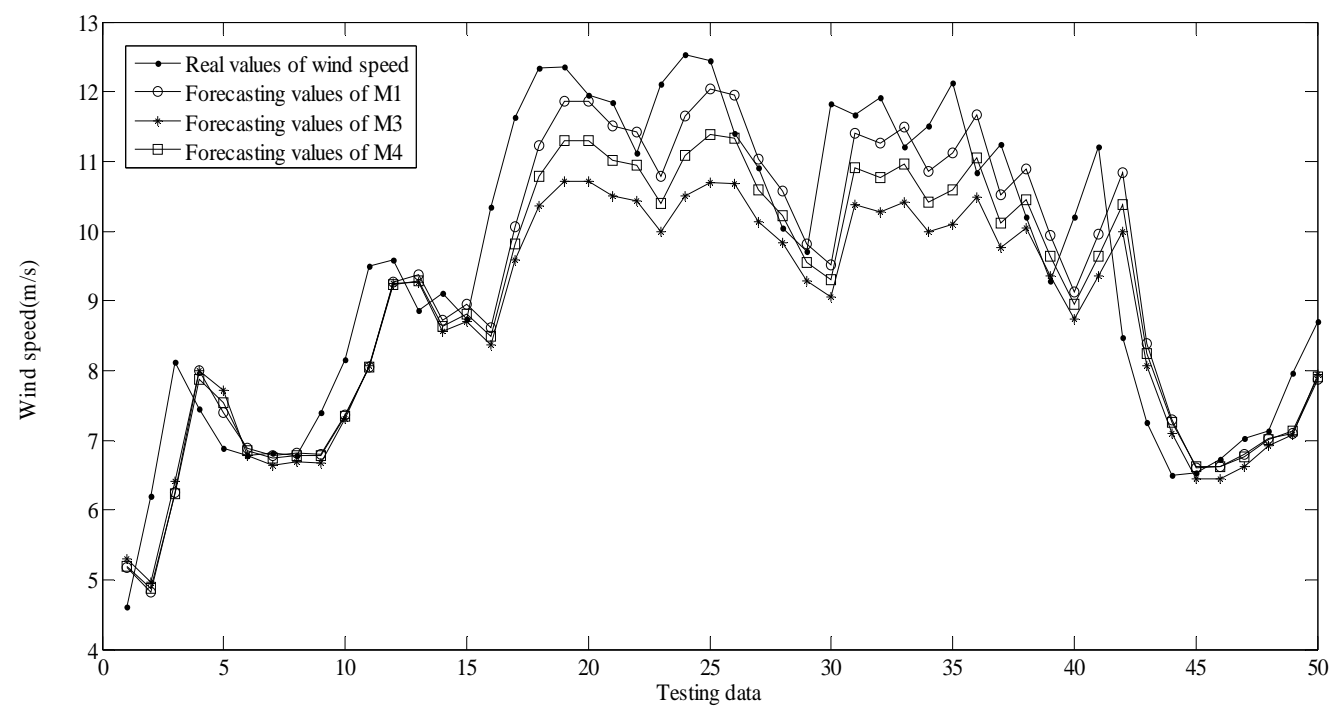

FIGURE II. FORECASTING VALUES OF THE THREE FORECASTING MODELS.

Different training samples may lead to different forecasting results. In order to get the best forecasting accuracy, different training sample sizes are used in the same testing sample. A conclusion can be drawn that it is very important to choose the proper training sample size during the modeling process.

In order to further test the performance of the proposed LSSVM model, the extensive simulation has been conducted. It shows that the applied model has a good performance on the error indexs such as MAE, MAPE, RMSE. Meantime, the training speed of the applied model is the fastest when comparing with the reference models. The excellent performance of the LSSVM model with coupled simulated annealing and simplex algorithm shows that the method has potential engineering values of wind speed forecasting and promotes the development of the wind power generation field.

\section{ACKNOWLEDGMENT}

This work was supported by National Natural Science Foundation of China (NSFC) (51277127).

\section{REFERENCES}

[1] D. Liu, D.X. Niu, H. Wang, and L. Fan, "Short-term wind speed forecasting using wavelet transform and support vector machines optimized by genetic algorithm,” Renew. Energy. vol. 62, pp. 592-597, February 2014.
[2] S. Salcedo-Sanz, E.G. Ortiz-Garcı, Á.M. Pérez-Bellido, A. PortillaFigueras, and L. Prieto, "Short term wind speed prediction based on evolutionary support vector regression algorithms,” Expert. Syst. Appl. vol. 38, pp. 4052-4057, April 2011.

[3] C. Zhang, Y. Liu, H. Zhang, and H. Huang, "Study on urban short-term gas load forecasting based on support vector machine model," J Tsinghua Univ. (Sci \& Technol.). vol. 54, pp. 320-325, March 2014.

[4] B.J. Chen, M.W. Chang, and C.J. Lin, "Load forecasting using support vector machines: A study on EUNITE competition 2001,” IEEE Trans. Power. Syst. vol. 19, pp. 1821-1830, November 2004.

[5] J. A. Suykens, and J. Vandewalle, "Least squares support vector machine classifiers,” Neural. Process. Lett. vol. 9, pp. 293-300, August 1998.

[6] H. Esen, F. Ozgen, M. Esen, and A. Sengur, "Modelling of a new solar air heater through least-squares support vector machines," Expert. Syst. Appl. vol. 36, pp. 10673-10682, September 2009.

[7] J. A. Suykens, T. Van Gestel, J. De Brabanter, B. De Moor and J. Vandewalle, Least Squares Support Vector Machines. World Scientific Publishing Co. Pte. Ltd, 2002, pp.98-100.

[8] G.Z. Li, W.Y. Liu, "Bus load short-term forecast based on LSSVM and Markov chain,” Power. Syst. Prot \& Control. vol. 38, pp. 55-66, June 2010.

[9] S. Xavier-de-souza, J.A. Suykens, J. Vandewalle, and D. Bollé, "Coupled simulated annealing," IEEE Trans. Syst. Man. Cy. B. vol. 40(2), pp. 320-335, April 2010.

[10] J.A. Nelder, and R. Mead, "A simplex method for function minimization,” Comput. J. vol. 7, pp. 308-313, 1965.

[11] X. K. Gao, C.A. Yao, X.C. Gao, and Y. Yu, "Identification of solar cell model parameters by combining analytical method with Nelder-Mead simplex method," Trans. CSAE, vol. 30, pp. 97-106, March 2014. 